\title{
Obtención de hidroxiapatita a través de residuos biológicos para injertos óseos dentales
}

\section{Obtaining hydroxiapatite through biological residues for dental bone graft}

Vanessa Sofía Bermúdez García ${ }^{1 d, a}$, Karina Huaman Mujica ${ }^{1, a}$, José Alberto Castañeda-Vía ${ }^{2, b}$, Carlos V. Landauro $^{3, c}$; Justiniano Quispe ${ }^{3, c}$; Lidia Yileng Tay Chu Jon ${ }^{1, e, d}$

\section{RESUMEN}

El objetivo de esta revisión de literatura es identificar las diferentes fuentes de obtención de hidroxiapatita (HAP) a partir de residuos naturales con la finalidad de utilizarla como injertos óseos dentales. La hidroxiapatita se puede clasificar según sus fuentes de origen en sintética o biológica; entre las fuentes de orígenes biológicos podemos encontrar de origen animal, origen acuático, origen coralino, origen de cáscara de huevo y de origen vegetal.Se describen los diferentes tipos de síntesis disponibles en la literatura para la obtención de hidroxiapatita a partir de cada fuente natural y su comportamiento como material de relleno óseo. Las fuentes naturales para obtención de hidroxiapatita a partir de residuos orgánicos reducen el impacto ambiental (bio basura) además de tener múltiples propiedades biocompatibles con el tejido óseo humano.

PALABRAS CLAVE: Trasplante óseo, durapatita, hidroxiapatita, residuo de alimentos.

Facultad de Estomatología,. Universidad Peruana Cayetano Heredia. Lima, Perú.

Escuela de Posgrado Víctor Alzamora Castro, Universidad Peruana Cayetano Heredia. Lima, Perú.

Facultad de Ciencias Físicas, Universidad Nacional Mayor de San Marcos, Lima, Perú.

Centro de Excelencia “Centro de Investigaciones Tecnológicas, Biomédicas y Medioambientales”. Bellavista, Callao, Perú.

Doctorando en Estomatología.

Magíster en Física con Mención en Física del Estado Sólido.

c Docente, Doctor en Física

d Investigador Principal

e Docente, $\mathrm{PhD}$ y Mg en Odontología, 


\section{SUMMARY}

The objective of this literature review is to identify the different sources for obtaining hydroxyapatite (HAP) from natural residues in order to use it as dental bone grafts. Hydroxyapatite can be classified according to its sources of origin as synthetic or biological; Among the sources of biological origins we can find of animal origin, aquatic origin, coral origin, egg shell origin and plant origin. The different types of synthesis available in the literature are described for obtaining hydroxyapatite from each natural source and its behavior as a bone filling material. The natural sources for obtaining hydroxyapatite from organic waste reduce the environmental impact (bio-waste) in addition to having multiple properties that are biocompatible with human bone tissue.

KEYWORDS: Bone transplantation, durapatite, hydroxyapatites, garbage.

\section{INTRODUCCIÓN}

La hidroxiapatita es el principal componente mineral del tejido óseo en los seres humanos, representando un depósito del $99 \%$ de calcio corporal, $80 \%$ del fósforo total del organismo y conformando el $96 \%$ del esmalte dental (1).

Al ser el principal componente mineral del tejido óseo, se convierte en un material biocompatible de primera elección para la reparación de este tejido duro, por sus múltiples propiedades, algunas de ellas son: biocompatibilidad, osteoconducción, propiedades mecánicas de rigidez y dureza.

Por estas características este material se viene utilizando en la industria dental como injertos óseos en defectos de rebordes alveolares desde los años 70’s.

Según su origen se puede obtener hidroxiapatita a partir de residuos animales y vegetales. Entre los de origen animal se pueden encontrar en restos óseos mamíferos, cáscara de huevo de aves, restos coralinos y en espinas y escamas de pescados.

Entre los residuos de origen vegetal podemos encontrar las algas marinas, hojas de albahaca, hojas de tréboles, hojas de té verde, bambú y madera de ratán.

La presente revisión de literatura tiene como objetivo clasificar la obtención de hidroxiapatita a partir de residuos naturales según su origen animal.

\section{HIDROXIAPATITA OBTENIDA DE RESIDUOS NATURALES}

Origen animal: En esta clasificación podemos encontrar residuos provenientes de diferentes especies como son: restos óseos mamíferos de diferentes especies, restos de cáscaras de huevo de aves, restos de escamas y espinas de animales acuáticos y restos de origen coralino.

\section{RESIDUOS PROVENIENTES DE RESTOS ÓSEOS MAMÍFEROS}

Bovino. - La HAP de origen bovino se emplea hace más de 60 años y se obtiene del hueso fémur. El procedimiento usual de síntesis es el de llevar a calcinación los huesos a temperaturas hasta $700{ }^{\circ} \mathrm{C}$ para obtener una HAP pura y con partículas esféricas, como lo hicieron Londoño-Restrepo y col. (2). Se ha demostrado que la HAP bovina formando compuestos muestra buena viabilidad celular en condiciones invitro, como lo hicieron Sun y col. sintetizando compuestos HAP/colágeno (3). El xenoinjerto con más estudios clínicos in-vitro e in-vivo son los de origen bovino. El estudio clínico realizado por Rodríguez y Nowzari, ahonda sobre los riesgos y complicaciones asociados a estos materiales reportando 5 casos clínicos, con seguimiento por periodos de entre 2 y 13 años, en los que encontraron que el xenoinjerto implantado con el tiempo no es biodegradable, sin embargo, podrían darse reacciones inmunes agudas y crónicas que a largo plazo se solucionan con el retiro del injerto óseo (4). 
Porcino. - La HAP de origen porcino se obtiene de hueso cortical del cerdo. La fase inorgánica de este hueso está compuesta por hidroxiapatita mientras que la fase orgánica está compuesta por colágeno y proteínas; esta fase orgánica está encargada de la flexibilidad, resistencia y elasticidad del cuerpo (5). Por medio de procesos hidrotermales se procede con la eliminación de este componente quedando sólo la parte inorgánica. El procedimiento seguido por Ramírez-Gutiérrez y col. consiste en calcinar el hueso porcino a temperaturas de $600{ }^{\circ} \mathrm{C}$ y $1000{ }^{\circ} \mathrm{C}$ luego del procedimiento hidrotermal, encontrando para ambos casos una HAP cristalina con la diferencia de que presenta residuos de $\mathrm{MgO}$ cuando se somete a 1000 ${ }^{\circ} \mathrm{C}$ (6). Así mismo, el estudio de Salamanca y col. muestra que el injerto porcino proporciona viabilidad celular similar a los osteoblastos y que es un sustituto óseo potencial en la aplicación clínica (7).

Camélido: La muestra de esta especie, se obtiene también de la parte cortical del hueso fémur de camello. Luego de pasar por el proceso químico de limpieza y desproteinización se procede con la síntesis de HAP mediante el método de calcinación a $900{ }^{\circ} \mathrm{C}$ que da como subproductos: fosfato de calcio, sodio, magnesio, hierro, aluminio y zinc, la presencia de estos oligoelementos en la HAP adquirida puede mejorar y acelerar el proceso de crecimiento óseo (5). En el estudio, realizado por Gashtasbi y col., se comparan injertos de hueso bovino e injertos de camélidos concluyendo que este último ofrece una respuesta celular favorable y similar regeneración ósea a la de un injerto bovino comercial (8).

Equino: La fuente de HAP se obtiene tanto de la columna como de la tibia del caballo, cuyas morfologías difieren en qué tan compacto o poroso es el hueso. Estos aspectos, además de los pre-tratamientos para la eliminación de la materia orgánica y la temperatura de calcinado fueron estudiados por Jang y col. en su búsqueda de HAP para aplicaciones biomédicas, encontrando que un hervor previo por 2 horas y un calcinado a $1300{ }^{\circ} \mathrm{C}$ por 12 horas son las condiciones ideales para la obtención de HAP, independientemente del tipo de hueso utilizado (9). Por su parte, Park y col. indicaron que luego de una extracción dental se mantuvo eficazmente las dimensiones óseas y se promovió la neoformación con la utilización de un injerto óseo derivado de caballo, esto debido a la estructura porosa que posee el injerto, la cual facilita la proliferación celular y por ende su actividad osteoconductora (10).

\section{RESIDUOS DE CÁSCARAS DE HUEVO DE AVES}

De gallina: La cáscara está compuesta entre un $96 \%$ y $98 \%$ de carbonato de calcio en forma de calcita y del $2 \%$ al $4 \%$ restante es una matriz orgánica que se localiza entre los cristales de carbonato cálcico (su espesor es de $0.3 \mathrm{~mm}$ ). El carbonato de calcio es precursor de HAP, por ello al pasar por diferentes procesos químicos y en adición de otros componentes que contengan iones fosfato, se llega a obtener el material del estudio. La síntesis de HAP a partir de este bio-desecho se puede realizar a través de la técnica más usada: el método sol-gel, previo proceso de calcinación a $900{ }^{\circ} \mathrm{C}$ para obtener hidróxido de calcio, que es soluble en agua y permite su uso en la técnica antes mencionada (11).

La hidroxiapatita derivada de cáscara de huevo de gallina ya se viene utilizando en estudios clínicos en seres humanos; el estudio revisado en esta literatura analiza la hidroxiapatita derivada de cáscara de huevo como sustituto óseo en la reparación de defectos óseos maxilares; donde injertan hidroxiapatita de cáscara de huevo en defectos periapicales luego de una apicectomía o luego de la extracción de un quiste residual en la zona del ápice radicular del maxilar. Dicho estudio se llevó a cabo en 8 pacientes adultos, con un grupo control cuyo injerto fue hidroxiapatita sintética comercial. Se realizó el seguimiento radiográfico semanal y se demostró que a las 8 semanas post injerto había una regeneración ósea significativa con un alto grado de densidad en la imagen radiográfica. El seguimiento fue de 3 meses, pero a los 2 meses se evidenció la regeneración ósea completa y con estudios celulares in vitro se evidencio la biocompatibilidad del compuesto. Este estudio concluye que la hidroxiapatita derivada de cáscara de huevo de gallina es superior en regeneración ósea en comparación con la hidroxiapatita sintética comercial (12).

El estudio de Park y col. se encargó de evaluar la cicatrización ósea de un injerto de hidroxiapatita de cáscara de huevo de gallina versus un injerto comercial bovino (Bi-oss-BO) en modelos animales, llegando a la conclusión que el producto de cáscara de 
huevo posee mejores propiedades osteoconductoras, con aumento de neo formación ósea y un alto grado de aposición ósea a las 8 semanas de injertado, esto debido a la estructura microporosa que posee y la biodegradabilidad en fluidos corporales que juegan un papel muy importante en la función de cicatrización ósea (13).

De avestruz: El avestruz es una especie nativa de África, país que alberga la mayor producción de esta ave, mientras que Brasil es el segundo país con la mayor población de avestruces en el mundo. Un avestruz hembra puede dar 60 huevos al año, un huevo de avestruz llega a pesar cerca de un kilo y medio; su cáscara está formada por $97.4 \%$ de carbonato de calcio, $1.9 \%$ fosfato de magnesio y $0.7 \%$ de fosfato tricálcico (14).

Teniendo en cuenta su composición, este bio desecho se vuelve útil en la síntesis de HAP que, al igual que con la cáscara de huevo de gallina, se procesa por precipitación química y calcinación (11). La diferencia entre el huevo de gallina y de avestruz radica principalmente en las porfirinas que constituyen su componente orgánica, la cual en el proceso de calcinación se transforma en $\mathrm{CO}_{2}$ gaseoso y no participa de la síntesis de HAP. (14)

El estudio de Durmus y col. evalúa la biocompatibilidad y actividad ósea de la hidroxiapatita tomada de cáscara de huevo de avestruz y la compara con una matriz ósea comercial, injertándolas en calotas de conejos adultos. Su evaluación fue realizada en 3 periodos de tiempo, 1 mes, 2 meses y 3 meses, concluyendo que la matriz ósea comercial tuvo mejor comportamiento en regeneración ósea y reabsorción, ya que al término de los 3 meses de evaluación la hidroxiapatita experimental de cáscara de avestruz aún no se había reabsorbido (15).

\section{RESIDUOS DE ANIMALES ACUÁTICOS}

\section{Espinas de pescado}

Desde el año 1995 se investigaron las propiedades físico químicas de las espinas de pescado, encontrando en ellas alto contenido de calcio, seguido de fósforo, sodio y magnesio. Según Pujie Shi y Meng Liu, dentro de su investigación, ellos concluyen que la fuente de espina de pescado es segura y presenta un bajo riesgo de transmisión de enfermedades; pero solo de algunas especies se puede llegar a obtener HAP, como son: el salmón, la carpa, la anchoa japonesa, la sardina, el blanquillo y el atún. A su vez, se ha investigado que solo algunasespecies presentan alto contenido de fosfato tricálcico (TCP) e hidroxiapatita como son: la anchoa japonesa, la sardina, la caballa, el blanquillo, la corvina, el pez ballesta, el pez lagarto, la caballa española, el pez volador, el congrio y el pez plano (16).

El método de síntesis gold estándar para esta especie es la calcinación entre $800^{\circ} \mathrm{C}$ a $1000^{\circ} \mathrm{C}$. En el estudio de Shi y col, muestra que la HAP obtenida de espinas de pescado (salmón) tienen un gran potencial regenerador óseo ya que contienen iones minerales esenciales (estroncio, magnesio y sodio), que conducen a la proliferación, diferenciación y adhesión célular para la formación de tejido mineralizado teniendo un mayor potencial de regeneración ósea en comparación con una hidroxiapatita sintética (17).

Otro estudios analizan la importancia de la hidroxiapatita derivada de espina de pescado, describiendo que el tamaño de los poros de este producto produce una interconectividad celular ideal, lo cual apoya la actividad biológica como el crecimiento y vascularización del tejido óseo. Las pruebas de bioactividad destacaron la proliferación de osteoblastos mostrando que los andamios producidos pueden moldearse rápidamente a las etapas de cicatrización ósea.

\section{Escamas de pescado}

Otra fuente natural encontrada en la bibliografía son las escamas de pescado, las cuales contienen material orgánico e inorgánico como colágeno e hidroxiapatita. Las investigaciones indican que las escamas de Tilapias son las ideales para la obtención de hidroxiapatita (17).

En el estudio de Pon y col., donde comparan hidroxiapatita obtenida de escamas de pescado versus hidroxiapatita sintética como material óseo regenerativo, se confirma que la hidroxiapatita derivada de escamas de pescado es biológicamente mejor que la hidroxiapatita sintética, además que tiene potencial uso como andamio óseo para la 
Tabla 1. Fuentes de obtención de hidroxiapatita, método de síntesis para aplicación como injerto óseo en odontología.

\begin{tabular}{|c|c|c|c|}
\hline \multirow{10}{*}{ 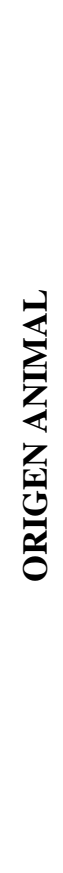 } & Residuo & Método de síntesis & Referencia \\
\hline & Restos óseos de Bovino & Calcinación $700^{\circ} \mathrm{C}-900^{\circ} \mathrm{C}$ & Khan - 2020 \\
\hline & Restos óseos de Porcino & Calcinación $600^{\circ} \mathrm{C}-1000^{\circ} \mathrm{C}$ & Ramirez-Gutierrez - 2017 \\
\hline & Restos óseos de Camélido & $\begin{array}{l}\text { Calcinación / tratamiento } \\
\text { termoquímico } 900^{\circ} \mathrm{C}\end{array}$ & Gashtasbi - 2020 \\
\hline & Restos óseos de Equino & Calcinación $1300^{\circ}$ & Jang - 2014 \\
\hline & $\begin{array}{l}\text { Residuos de cáscara de huevo de } \\
\text { Gallina }\end{array}$ & $\begin{array}{l}\text { sol-gel, previo proceso de } \\
\text { calcinación a } 900^{\circ} \mathrm{C}\end{array}$ & Arboleda - 2016 \\
\hline & $\begin{array}{l}\text { Residuos de cáscara de huevo de } \\
\text { Avestruz }\end{array}$ & precipitación química y calcinación & Malla - 2020 \\
\hline & $\begin{array}{l}\text { Residuos de espina de pescado (salmón, } \\
\text { carpa, anchoa japonesa, sardina, } \\
\text { blanquillo, atún) }\end{array}$ & Calcinación $800^{\circ} \mathrm{C}-1000^{\circ} \mathrm{C}$ & Shi - 2018 \\
\hline & $\begin{array}{l}\text { Residuos de escama de pescado } \\
\text { (tilapias) }\end{array}$ & Calcinación $800^{\circ} \mathrm{C}-1000^{\circ} \mathrm{C}$ & Huan -2011 \\
\hline & $\begin{array}{l}\text { Residuos de corales (ostras, mejillones, } \\
\text { almejas, estrella de mar) }\end{array}$ & sol-gel, calcinación e hidrotermal & Agbeboh - 2020 \\
\hline
\end{tabular}

neoformación celular (18). Por su parte, el estudio de Huang y col. confirma que la hidroxiapatita extraída de escamas de pescado es un biomaterial que presenta propiedades de morfología, ultraestructura y características bioquímicas muy similares al tejido óseo embrionario, siendo este producto prometedor para la fabricación de hueso artificial. (19)

\section{Residuos de origen coralino}

Desde 1996 se desarrollan las primeras investigaciones de obtención de hidroxiapatita a partir de corales marinos, su nombre científico es Goniopora $s p$. Entre estos corales se encuentran las ostras, los mejillones, las almejas y la estrella de mar, que dentro de su composición cuentan con $97 \%$ de carbonato de calcio en forma de fase aragonito, material que sirve de fuente de calcio como compuesto precursor en la preparación de HAP.

La síntesis de obtención de hidroxiapatita puede ser por precipitación sol-gel, calcinación e hidrotermal (11).
En un estudio de comparación de injertos óseos a base de hidroxiapatita tomado de corales para aplicaciones biomédicas, se comparó la hidroxiapatita sintética y la hidroxiapatita de coral en hueso mandibular con defectos periodontales, demostrando que la hidroxiapatita coralina tiene propiedades de neoformación ósea, rápida vascularización y es bioreabsorbible, esto debido a su alta porosidad que permiten la unión ósea y la fijación de injertos (20). En conclusión, se encuentra en la literatura múltiples fuentes naturales de origen animal para la obtención de hidroxiapatita a partir de residuos orgánicos de forma eco amigable (tabla $\mathrm{N}^{\circ}$ 1), a bajo costo, reduciendo el impacto ambiental (bio basura), siendo sus ventajas: biocompatibilidad con el tejido óseo humano, estimula la osteoinducción y osteoconducción, aumenta la neo formación ósea con un alto grado de aposición ósea, regeneración y cicatrización ósea acelerada $(21,22,23)$. 


\section{Correspondencia:}

Vanessa Bermudez García

Av. Honorio Delgado 430, San Martín de Porres

15102

Universidad Peruana Cayetano Heredia - Campus

Central

Correo electrónico: vanessa.bermudez@upch.pe

\section{REFERENCIAS BIBLIOGRÁFICAS}

1. Connie M, Weaver MP. Calcium. Advances in Nutrition. 2011; 2 (3): 290-2.

2 Londoño-Restrepo SM, Jeronimo-Cruz R, RubioRosas E, Rodriguez - García M.E. The effect of cyclic heat treatment on the physicochemical properties of bio hydroxyapatite from bovine bone. J Mater Sci: Mater Med. 2018;29(52).

3. Rui-Xue S, Yao LV, Yu-Rong N, et al. Physicochemical and biological properties of bovine-derived porous hydroxyapatite/collagen composite and its hydroxyapatite powders. Ceramics International 2017; 43(18): 16792-98.

4. Rodríguez AE, Nowzari $\mathrm{H}$. The long-term risks and complications of bovine-derived xenografts. Rev ADM. 2020;77(2):108-116.

5. Nannmark U, Sennerby L. The bone tissue responses to prehydrated and collagenated cortico-cancellous porcine bone grafts: a study in rabbit maxillary defects. Clin Implant Dent Relat Res. 2008;10(4):264-70.

6. Ramirez-Gutierrez CF, Londoño-Restrepo SM, Del Real A, Mondragón M, Rodriguez-García ME. Effect of the temperature and sintering time on the thermal, structural, morphological, and vibrational properties of hydroxyapatite derived from pig bone. Ceramics International 2017;43,10: 7552-59.

7. Salamanca E, $\underline{\mathrm{Hsu}} \mathrm{Ch}$, Huang HM, et al. Bone regeneration using a porcine bone substitute collagen composite in vitro and in vivo. Sci Rep. 2018;8(1):984.

8. Gashtasbi F, Hasannia S, Hasannia S, et al. Comparative study of impact of animal source on physical, structural, and biological properties of bone xenograft. Xenotransplantation. 2020: e12628.

9. Jang K-J, Cho WJ, Seonwoo H, et al. Development and Characterization of Horse Bone-derived Natural Calcium Phosphate Powders. Journal of Biosystems Engineering. 2014;39(2):122-33.

10. Park J, Koo Y, Kim KT, et al. Socket preservation using deproteinized horse-derived bone mineral. J Periodontal Implant Sci. 2010;40(5): 227-31.

11. Monroe EA, Votava W, Bass DB, McMullen J. New calcium phosphate ceramic material for bone and tooth implants. Journal of dental research. 1971;50(4): 86061.
12. Kattimani VS, Chakravarthi PS, Kanumuru NR, et al. Eggshell derived hydroxyapatite as bone graft substitute in the healing of maxillary cystic bone defects: a preliminary report. J Int Oral Health. 2014;6(3):1519.

13. Park JW, Bae SR, Suh JY, et al.. Evaluation of bone healing with eggshell-derived bone graft substitutes in rat calvaria: A pilot study. J Biomed Mater Res. 2008; 87: 203-14.

14. Ferreira JRM, Louro LHL, Costa AM, De Campos JB, Prado da Silva MH. Ostrich eggshell as calcium source for the synthesis of hydroxyapatite and hydroxyapatite partially substituted with zinc. Cerâmica. 2016; 62(364), 386-91.

15. Durmus E, Celik I, Aydin M, Yildirim G, Sur E. Evaluation of the Biocompatibility and osteoproductive activity of ostrich eggshell powder in experimentally induced calvarial defects in rabbits. Journal of biomedical materials research. 2008; 86. 82-9.

16. Pujie S, Meng L, Fengjiao F, Cuiping Y, Weihong L, Ming D. Characterization of natural hydroxyapatite originated from fish bone and its biocompatibility with osteoblasts, Materials Science and Engineering. 2018; 90: 706-712.

17. Shi P, Liu M, Fengjiao F, Cuiping Y, Lu W, Du M. Characterization of natural hydroxyapatite originated from fish bone and its biocompatibility with osteoblasts. Materials Science and Engineering. 2018; 90: 706-12.

18. Pon-On W, Suntornsaratoon P, Charoenphandhu N, Thongbunchoo J, Krishnamra N, Tang IM. Hydroxyapatite from fish scale for potential use as bone scaffold or regenerative material. Mater Sci Eng C Mater Biol Appl. 2016;62:183-189.

19. Huan Y-C, Hsiao P-C, Chai H-J. Hydroxyapatite extracted from fish scale: Effects on MG63 osteoblastlike cells. Ceramics International. 2011;(37): 18251831.

20. Malla K, Regmi S, Nepal A, et al. Extraction and Characterization of Novel Natural Hydroxyapatite Bioceramic by Thermal Decomposition of Waste Ostrich Bone. International Journal of Biomaterials. 2020; 1: 1-10.

21. Gashtasbi F, Hasannia S, Hasannia S, et al. Comparative study of impact of animal source on physical, structural, and biological properties of bone xenograft. Xenotransplantation. 2020: e12628.

22 Agbeboh NI, Oladele IO, Daramola OO, Adediran AA, Olasukanmi OO, Tanimola MO. Environmentally sustainable processes for the synthesis of hydroxyapatite. Heliyon. 2020;6(4):e03765.

23. Huang J, Ratnayake J, Ramesh N, Dias G.J. Development and characterization of a biocomposite material from Chitosan and New Zealand-Sourced Bovine-Derived Hydroxyapatite for Bone Regeneration. ACS omega 2020; 5,27: 16537-46. 
Recibido : 29-01-2021

Aceptado : 22-04-2021 